Review

\title{
Designing Nanoparticle-based Drug Delivery Systems for Precision Medicine
}

\author{
Jianhua Yang1, Chengyou Jia², Jianshe Yang2,3四 \\ 1. Department of Orthopaedics, Longgang District People's Hospital, Shenzhen 518172, China \\ 2. Shanghai Research Center for Thyroid Diseases, Shanghai Tenth People's Hospital, Tongji University School of Medicine, Shanghai 200072, China. \\ 3. Health and Life Science College, the Chinese University of Hong Kong, Shenzhen 518172, China. \\ $\triangle$ Corresponding author: Professor Jianshe Yang, Shanghai Research Center for Thyroid Diseases, Shanghai Tenth People's Hospital, Tongji University School \\ of Medicine, Shanghai 200072, China. Tel: +86-21-66302721, E-mail: yangjs@impcas.ac.cn.
}

(c) The author(s). This is an open access article distributed under the terms of the Creative Commons Attribution License (https://creativecommons.org/licenses/by/4.0/). See http://ivyspring.com/terms for full terms and conditions.

Received: 2021.03.25; Accepted: 2021.05.25; Published: 2021.06.05

\begin{abstract}
Traditional drugs are facing bottlenecks of lower solubility, absorption, and especially the inefficient organs or cells targeting during the precision medicine era. It is urgently needed to discover and establish new methods or strategies to modify old drugs or create new ones against the above defects. With the support of nanotechnology, the solubility, absorption and targeting of traditional drugs were greatly improved by modifying and fabricating with various types of nanoparticles to some extent, though many shortages remain. In this mini-review we will focus on advances in several most commonly used nanoparticles, from their nature and design, to drug delivery system and clinical application, that they overcome heterogeneous barriers in precision medicine, thereby ultimately improve patient outcome overall.
\end{abstract}

Key words: nanoparticles, drug delivery system, precision medicine, drug design

\section{Introduction}

From a scientific perspective we know the engineering nanomaterials have significant priority to promoting disease diagnosis and treatment. Nanotechnology can facilitate the drug delivery through nanomaterials targeted modification, transport of desired molecules to specific organelles. The nanotechnology program has thrived worldwide since 20 years ago, such as the US National Science and Technology Council (NSTC) launched the National Nanotechnology Initiative (NNI) in 2000, and presented clear plans and major challenges in this field. Nanoparticles (NPs) occupy a large part of all the plans with the source variation and preparation diversity. What-forever, the improvement in stability and solubility of the NPs depends on many factors including the NP composition, formulation, chemical structures of the small drug molecules and its carriers, temperature, $\mathrm{pH}$, etc., thus improving the safety and effectiveness [1, 2]. Owning these priorities, NPs have been widely used and produced promising results both in pre-clinical and clinical stages $[2-5,7,8]$. As nanoparticle-based precision therapies are applied in a broad area, such as cancer therapy, immunotherapy, and recently in virus infected disease, a full span and timely understanding of NPs progress is particularly important. Here we will report and review several most commonly used NPs in the terms of its preparation and precision medicine application, and expect to afford the guidance to optimize the strategies from its design to clinical application.

\section{Lipid-based NPs}

Lipid-based NPs can be designed into various subpopulation structures, among them the spherical structures is most typical. This type of lipid-based NPs is constituted of at least one lipid bilayer surrounding an interior water chamber (Figure 1) [4]. Also the lipid-based nano-micelles is of well promise. Lipid-based NPs is regarded as an important drug delivery system relying on its sorts of advantages: simple formulation, self-assembly, well biocompatibility and high bioavailability, large 
payloads, and improvable physicochemical properties utilized to regulate their biological applications. [5] Thus the lipid-based NPs is the most frequent selection among FDA approved nanomedicine and other countries worldwide [6, 7].

Liposome is one of the most typical lipid NPs subsets, which are the monolayer and multilayer vesicles composed of phospholipids. This special structure allows liposomes convenient to carry and deliver drugs with difference characters from hydrophilic, hydrophobic to lipophilic, and they can even adsorb hydrophilic and lipophilic compounds simultaneously, thus making it more versatile [8]. The stability of liposomes whether in vitro or in vivo is influenced by NPs size, surface charge, lipid component, and surface modification (ligand or polymer), respectively [9]. Liposomes are usually modified on its surface to expand their fluidity and enhance drug delivery, and targeting as well. Unmodified liposomes are cleared by RES, while the surface modifications help to avoid the RES and thus prolongs the blood circulation time [10].

Another notable lipid-based NPs subgroup is the lipid nanoparticles widely used for nucleic acid delivery (lipid nanoparticles, LNPs). The micelle structures mode makes LNPs different from traditional liposomes. This formation is within the particle core but not outer, and its morphology can be controlled and modulated according to the formulation and synthesis parameters, and the special formulation and morphology of these LNPs make its with serum stability and could not create compounds with human serum albumin [11]. There are commonly four main LNPs components: cationic or ionizable lipids, phospholipids, cholesterol, and polyethylene glycol lipids [12-14]. For LNPs, the effectiveness of nucleic acid delivery and its easy synthesis procedure, small volume (0.5-1 micron), and stability in circulation system make it very important to personalized gene therapy applications. The ionizable LNPs are ideal carriers in nucleic acid therapies because they are near neutral charges at physiological conditions, whereas charges in acidic inclusions can facilitate the escape to intracellular release [15].

Nevertheless, despite these advantages, the LNPs system is still limited due to the low drug load and biodistribution, leading to high uptake in the liver and spleen, and result in the acute cumulative drug injury. Improving the drug load capability and targeted uptake are deserved to be resolved immediately.

\section{Polymer NPs}

Polymer NPs can be synthesized using natural or synthetic materials and form various possible structures and features (Figure 1). A variety of techniques were used for the synthesis of polymer NPs, such as emulsification (solvent replacement or diffusion), nano-precipitation, ionic gelation, and microfluidics, all of which yielded different end products [16, 17]. Polymer NPs also have variable drug delivery capabilities which depends where and how does the drugs combine with NPs, for instance, drugs can be encapsulated within the NPs nucleus, embedded into the polymer matrix, bound to the polymer through chemical reactions or bonded to the NPs surface. Polymer NPs is an ideal material for drug co-delivery. The loaded drugs can be hydrophobic and hydrophilic compounds or other small molecules and biological macromolecules, such as RNAs/DNAs, proteins and anticancer drug paclitaxel $[17,18]$. By regulating the chemical and physical properties of NPs and drug can realize the accurate control of the loading effect and release kinetics [18].

There are two usual NPs polymers forms: nanocapsules (cavities surrounded by polymer membranes or enclosures) and nanospheres (nanospheres, solid matrix systems). NPs can be further divided into polymeric vesicles, micelles and dendrimers.
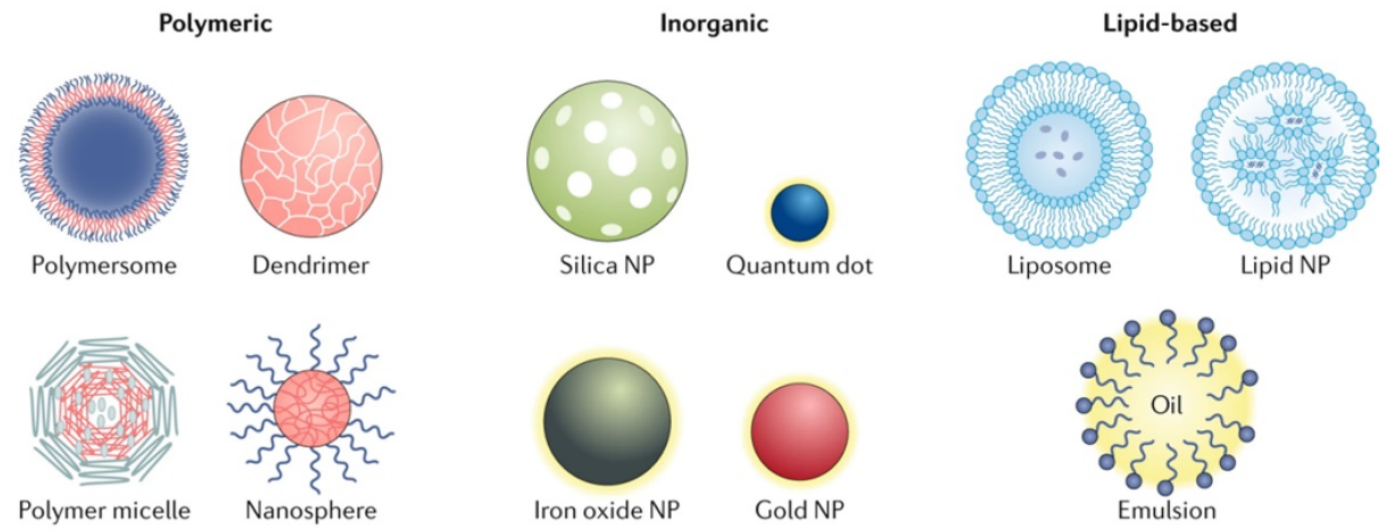

Figure 1. Different types of the nanoparticles. NP-based drug delivery systems can be classified into different types including polymeric, inorganic and lipid-based vehicles [4]. 
Polymeric vesicles are similar with liposomes, but more stable and efficient for drug retention due to its relative closed structure, thus they are more effective to deliver therapeutic drugs to the cytoplasm [19]. Polymeric vesicles commonly used for these uses include polyethylene glycol (PEG) and polydimethylsiloxane (PDMS).

Micelles are the nanospheres with hydrophilic inner core surrounded by hydrophobic coating through polymer NPs self-assembling, which can protect the transport of waterborne drugs and improve the cycling time. This type nanosphere can load various drugs, especially some biommolecules used in clinical trials of cancer therapy [20].

Dendrimers is a highly dendritic polymer with complex three-dimensional structures with highly controlled physical and chemical parameters. The active functional groups outside the dendrimers are prone to be fabricated with biomolecules with targeted tendency, while therapeutic drugs can be loaded inside. The loading capacity of dendrimers is promising, whereas research interest is focusing on delivering the RNA, DNA and proteins. In these applications, some charged polymers, such as polyethylenimine (PEI) and polyaminoines (PAM), are commonly used. Some clinical trials currently ondergoing just select several tree-like polymer products (described in reference 21 and 22) as test materials which owning the anti-inflammatory, transfection functions, also they are used as topical gels and contrast agents [21,22].

Overall, polymer NPs is an ideal drug delivery material due to its biodegradability, water solubility, biocompatibility, biomimeticity and storage stability. Their all-round surfaces targeted modifications enable them to transport diverse materials to the target tissue and play an important role for diagnosis and treatment in precision medicine. However, due to the high degree of polymerization of polymer NPs, some disadvantages will lead to the risk of particle aggregation and toxicity, fewer polymer nanopharmaceuticals have the clinical license by FDA, only a large number of clinical trials are permitted [23].

\section{Inorganic NPs}

To date, some inorganic materials are prepared into nanostructured materials by monomer or by chemical doping with other matrix for application on drug delivery and imaging. The inorganic nanomaterials are designed into various sizes, structures and geometries. Among them, gold nanoparticles (AuNPs) are of the most frequently been studied with its different form like nanospheres and nanorods, nanoshells [24]. Furthermore, the unique electrical, magnetic and optical nature of inorganic NPs (such as Au NPs, quantum dots and iron oxide), decided by the substrate properties, endowing them with many potential effects, like photothermal transforming effect to be a promising application for thermal-therapy [25].

Iron oxide is another common inorganic NPs and accounts for the vast majority of clinical studies in the world, including prognostic imaging and nano-based raditherapy for metabolic diseases and cancers. The magnetic iron oxide NPs consists of $\mathrm{Fe}_{3} \mathrm{O}_{4}$ or $\mathrm{Fe}_{2} \mathrm{O}_{3}$ with superparamagnetic properties in some sizes and has been successfully applied in contrast agents, drug delivery carriers, and therapy [26, 27]. Calcium phosphate and mesoporous silica are the other important inorganic NPs used to transport DNA/ RNA or drugs [28].

These types of inorganic NPs have unique magnetic, radioactive and plasmonic properties, which make them qualified for clinical diagnosis, imaging, and photothermal therapy. Most of them have good biocompatibility and stability. Cell and animal experiments have demonstrated these metal cores of NPs of very low cytotoxicity, and kept stable status during the course of intake and targeting to the organs with few interactions with surroundings. While, essential effort should be made to promote the solubility and reduce toxicity of these materials, especially when the heavy metals were enrolled into preparation system.

\section{Carbon NPs}

Carbon nano-materials include a various types of which are containing sole carbon skeleton, such as graphene, fullerene and the most used carbon nanotubes. Due to the rigid structure and low water-solubility, these materials have ever been neglected for biological application. While the unique chemical and physical characteristics of these materials presented after a series of special modification. In our previous studies, we explored the potential value of carbon nanotubes for its radiosensitizer role [29], and a surprised effect for inhibiting the prostate cancer cells without depending on the hormone-deprived therapy [30], even in the novo antiviral nano-medicine intermediate for SARS-CoV-2 prevention (Figure 2) [31].

\section{NPs in precision medicine}

The term "precision medicine" has evolved from "personalized medicine", which is based on the specific disease with individual unique genetic and molecular profile referred to a fully personalized treatment plan [32]. Years after the Human Genome Project finished, we consider prudently that 
"personalized" is too ambitious and restrictive, and find a substantial amount of relevant information, notable clinical parameters and environmental factors are difficult to be counted by this word anymore. However, the term "precision medicine" greatly expands the practical meaning which including the development of specific drugs and medical practice to a well-defined groups of individuals, both on their susceptibility to a particular disease and probability of responding to a given treatment plan. Consequentely any intervention including primary preventive and comprehensive treatments could be used to the specific subpopulations and they will definitely benefit from these targeted intervention, resulting in maintaining the resources management and systemic equities at high level as possible [33, 34].

Precision medicine can improve the clinical treatment method to minimize the limitations of the conventional therapies. For example, the biomarkers identification and concomitant diagnostics have been a standard in oncology diagnostics and treatments. Without the aidance of these techniques, anti-cancer nanopharmaceuticals will be difficult to work [35].

NPs designed for specific patient groups can break the limitations of current drug delivery, absorption and metabolism, and ultimately qualify more patients get benefit from precision medicine [36].

Applications of nanomaterials in precision medicine have emerged since the launch of the Precision Medicine Initiative (PMI) in 2015 [37]. An early clinical detection for personalized biomolecular adsorbed on graphene oxide nanosheets was exerted with pancreatic cancer patient blood in 2019 [38]. Graphene oxide can combine the unique characteristics of a small amount of albumin to enable strong adsorption of low-level proteins present in plasma. Other magnetic NPs or AuNPs showed the priority in biomarker detection analysis with much less time and consumes [39].

Beside the diagnostic usage, some of NPs can reshape the tumor microenvironment, thereby enhancing the sensitivity of tumors to specific treatments [40]. Endothelial cells of tumors can be manipulated by the microRNA delivered by NPs, therefore alters the vascular system microenvironment and makes the tumor much more sensitive to conventional cancer therapies. Similar bioinspired lipoproteins have been used to reshape tumors and increase NPs accessibility to cancer cells by 27 times [41]. The use of photothermal NPs can improve the wettability and anti-solid tumor activity of CAR-T cells [42] or lead the direct physical destroy to tumors or viruses [30]. NPs can also regulate immune function and make cancer cells sensitive to treatment, homogenize environments, and make more patients respond to accurate treatment [43].

Based on the above description, in Table 1, some examples of nanoparticle-based drug delivery systems that are on market or in clinical trials have been presented, like COVID-19 vaccine, DaunoXome, Doxil, Feraheme, etc., along with their delivery systems (liposomes, polymers, metals, or others), active ingredients, target diseases' names, and so on.
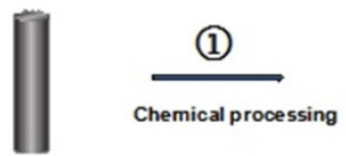

MWCNT

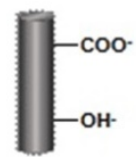

p-CNTS

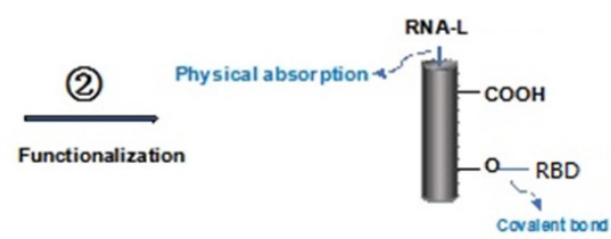

f-WCNTS

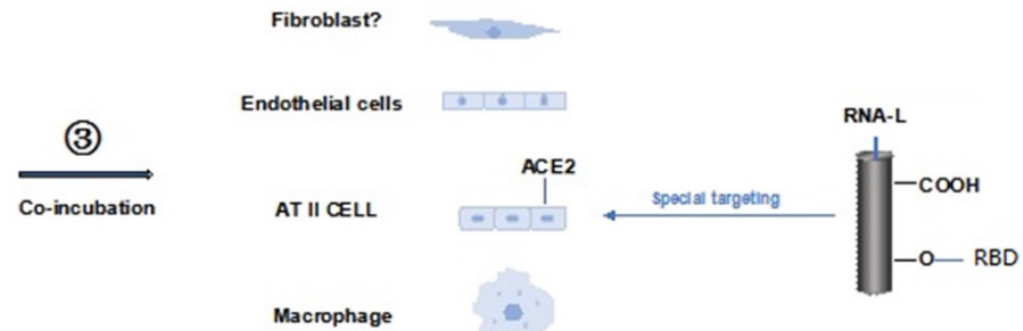

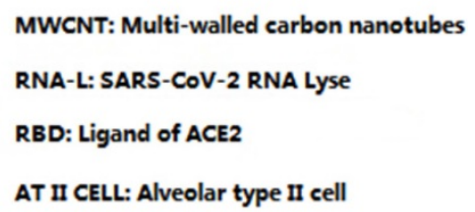

MWCNT: Multi-walled carbon nanotubes

RBD: Ligand of ACE2

AT II CELL: Alveolar type II cell

Figure 2. Flow chart of preparation of functional multi-walled carbon nanotubes. This flow chart described briefly the MWCNT was fabricated with strong acid and base conditional mixture in order to achieve the p-WCNT (chemical process); then modified with RNA layse and receptor binding domain (RBD) by covalent conjugation and physical absorption to get f-WCNT (functionalization); thereafter, f-WCNT was used in the multi-cell culture system interacting with SARS-CoV-2 to identify the special affinity of f-WCNT to ACE2 labeled alveolar type II cells and the inhibition capacity to SARS-CoV-2 [31]. 
Table 1. Some examples of NPs applied in clinic and clinical trials

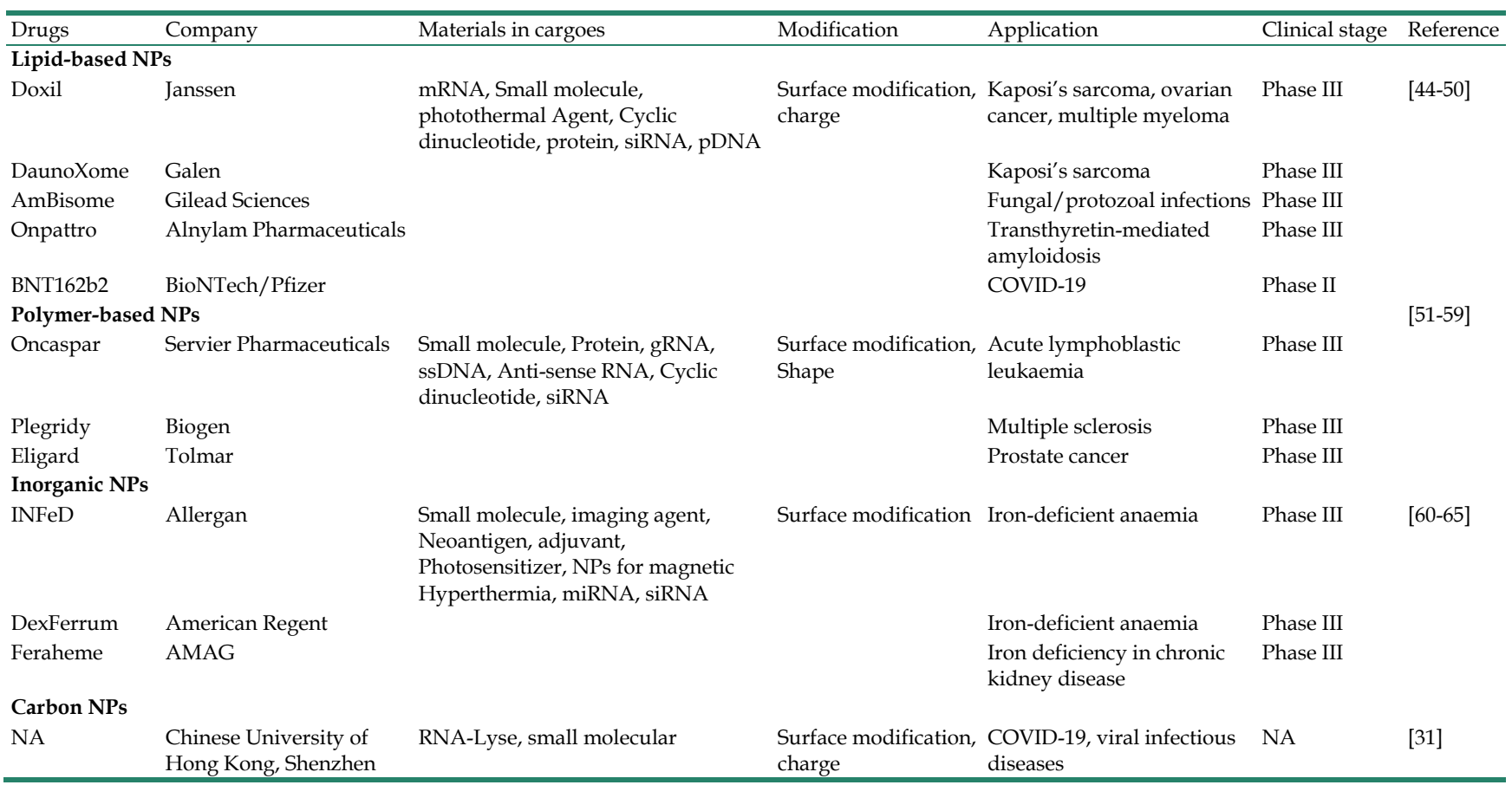

\section{Conclusions}

To sum up, the combination of NPs and precision medicine promotes the development of these two fields. Accurate medical classification of patients can accelerate the clinical transformation of many NPs developed for specific patients. Conversely, NPs by improving the delivery and efficacy of precision drugs, more patients are included in the classified population, thus increasing the success rate of precision medicine and benefiting patients. Application of NPs for precision medicine is of precisely design and control. The carefully designed method was able to tune the pharmacokinetics of the therapeutic drug to meet the requirements of solubility, administration or biodistribution and has been successful in the study. Yet the three challenges still need to be solved: (1) detailed and optimized synthesis process for controllable and renewable nanoparticle; (2) found the standard system for clinical evaluation of NPs; (3) implementation of good manufacturing practices (GMP), develop a practical clinical transition plan to achieve mass production.

\section{Acknowledgements}

\section{Funding}

This work was supported by the Special Research Project of Third Affiliated Hospital, the Chinese University of Hong Kong, Shenzhen, (Grant No. SLY-2019-01).

\section{Competing Interests}

The authors have declared that no competing interest exists.

\section{References}

1. Kou L, Bhutia YD, Yao Q, et al. Transporter-guided delivery of nanoparticles to improve drug permeation across cellular barriers and drug exposure to selective cell types. Front Pharmacol. 2018;9:27.

2. Blanco E, Shen H, Ferrari M. Principles of nanoparticle design for overcoming biological barriers to drug delivery. Nat Biotechnol. 2015;33(9):941-951.

3. Mitragotri S, Lammers T, Bae YH, et al. Drug delivery research for the future: Expanding the nano horizons and beyond. J Control Release. 2017;246:183-184.

4. Mitchell MJ, Billingsley MM, Haley RM, et al. Engineering precision nanoparticles for drug delivery. Nat Rev Drug Discov. 2021;20(2):101-124.

5. Sercombe L, Veerati T, Moheimani F, et al. Advances and challenges of liposome assisted drug delivery. Front Pharmacol. 2015;6:286.

6. Anselmo AC, Mitragotri S. Nanoparticles in the clinic: an update. Bioeng Transl Med. 2019;4(3):e10143.

7. Caliceti P, Matricardi P. Advances in drug delivery and biomaterials: Facts and vision. Pharmaceutics. 2019;11(1):48.

8. Teymouri M, Mashreghi M, Saburi E, et al. The trip of a drug inside the body: From a lipid-based nanocarrier to a target cell. J Control Release. 2019;309:59-71.

9. Lalloz A, Bolzinger MA, Faivre J, et al. Effect of surface chemistry of polymeric nanoparticles on cutaneous penetration of cholecalciferol. Int J Pharm. 2018;553(1-2):120-131.

10. Wang SJ, Huang WS, Chuang CM, et al. A phase 0 study of the pharmacokinetics, biodistribution, and dosimetry of ${ }^{188} \mathrm{Re}$-liposome in patients with metastatic tumors. EJNMMI Res. 2019;9(1):46.

11. Piazzini V, D'Ambrosio M, Luceri C, et al. Formulation of nanomicelles to improve the solubility and the oral absorption of silymarin. Molecules. 2019;24(9):1688

12. Viger-Gravel J, Schantz A, Pinon AC, et al. Structure of lipid nanoparticles containing siRNA or mRNA by dynamic nuclear polarization-enhanced NMR spectroscopy. J Phys Chem B. 2018;122(7):2073-2081.

13. Li X, Nakayama K, Goto T, et al. Comparative evaluation of the extraction and analysis of urinary phospholipids and lysophospholipids using MALDI-TOF/MS. Chem Phys Lipids. 2019;223:104787. 
14. Gilbile D, Docto D, Kingi DT,et al. How well can you tailor the charge of lipid vesicles? Langmuir. 2019;35(48):15960-15969.

15. Suzuki Y, Hyodo K, Suzuki T, et al. Biodegradable lipid nanoparticles induce a prolonged RNA interference-mediated protein knockdown and show rapid hepatic clearance in mice and nonhuman primates. Int J Pharm. 2017;519(1-2):34-43.

16. Kurd M, Sadegh Malvajerd S, Rezaee S, et al. Oral delivery of indinavir using mPEG-PCL nanoparticles: preparation, optimization, cellular uptake, transport and pharmacokinetic evaluation. Artif Cells Nanomed Biotechnol. 2019;47(1):2123-2133.

17. Sawasdee K, Sucharitakul J, Dhammaraj T, et al. Encapsulation of the reductase component of p-hydroxyphenylacetate hydroxylase in poly(lactide-co-glycolide) nanoparticles by three different emulsification techniques. IET Nanobiotechnol. 2018;12(4):423-428.

18. Lim EB, Vy TA, Lee SW. Comparative release kinetics of small drugs (ibuprofen and acetaminophen) from multifunctional mesoporous silica nanoparticles. J Mater Chem B. 2020;8(10):2096-2106.

19. Araste F, Aliabadi A, Abnous K, et al. Self-assembled polymeric vesicles: Focus on polymersomes in cancer treatment. J Control Release. 2021;330:502-528.

20. Pérez-Herrero E, Fernández-Medarde A. Advanced targeted therapies in cancer: Drug nanocarriers, the future of chemotherapy. Eur J Pharm Biopharm. 2015;93:52-79.

21. Maciel D, Guerrero-Beltrán C, Ceña-Diez R, et al. New anionic poly(alkylideneamine) dendrimers as microbicide agents against HIV-1 infection. Nanoscale. 2019;11(19):9679-9690.

22. Ziemba B, Sikorska H, Jander M, et al. Anti-tumour activity of glycodendrimer nanoparticles in a subcutaneous MEC-1 xenograft model of human chronic lymphocytic leukemia. Anticancer Agents Med Chem. 2020;20(3):325-334.

23. Caster JM, Patel AN, Zhang T, et al. Investigational nanomedicines in 2016: a review of nanotherapeutics currently undergoing clinical trials. Wiley Interdiscip Rev Nanomed Nanobiotechnol. 2017;9:1416.

24. Darweesh RS, Ayoub NM, Nazzal S. Gold nanoparticles and angiogenesis: molecular mechanisms and biomedical applications. Int J Nanomed. 2019;14:7643-7663.

25. Zhang D, Wu T, Qin X, et al. Intracellularly generated immunological gold nanoparticles for combinatorial photothermal therapy and immunotherapy against tumor. Nano Lett. 2019;19(9):6635-6646.

26. Wu L, Zhang F, Wei Z, et al. Magnetic delivery of $\mathrm{Fe}_{3} \mathrm{O}_{4} @$ polydopamine nanoparticle-loaded natural killer cells suggest a promising anticancer treatment. Biomater Sci. 2018;6(10):2714-2725.

27. Liang J, Yang $X$, Liu D, et al. Lipid/hyaluronic acid-coated doxorubicin- $\mathrm{Fe}_{3} \mathrm{O}_{4}$ as a dual-targeting nanoparticle for enhanced cancer therapy. AAPS PharmSciTech. 2020;21(6):235

28. Dong $\mathrm{Y}$, Liao $\mathrm{H}, \mathrm{Yu}$, et al. Incorporation of drug efflux inhibitor and chemotherapeutic agent into an inorganic/organic platform for the effective treatment of multidrug resistant breast cancer. J Nanobiotechnol. 2019;17(1):125.

29. Yang J, Li W, Li Q, et al. Oxygen adsorption by carbon nanotubes and its application in radiotherapy. IET Nanobiotechnol. 2007;1(1):10-14.

30. Yu BZ, Yang JS, Li WX. In vitro capability of multi-walled carbon nanotubes modified with gonadotrophin releasing hormone on killing cancer cells. Carbon. 2007; 45(10): 1921-1927.

31. Yang J. Inhibition of SARS-CoV-2 replication by acidizing and RNA lyase-modified carbon nanotubes combined with photodynamic thermal effect. J Explor Res Pharmacol. 2020;5(2):18-23.

32. Jain KK. Personalized medicine. Curr Opin Mol Ther. 2002;4:548-558.

33. National Research Council (US) Committee on a framework for developing a new taxonomy of disease. Toward precision medicine: building a knowledge network for biomedical research and a new taxonomy of disease. Washington (DC), USA: National Academies Press. 2011.

34. Khoury MJ, Galea S. Will precision medicine improve population health? JAMA. 2016;316:1357-1358.

35. Jiang M, Yang J, Li K, et al. Insights into the theranostic value of precision medicine on advanced radiotherapy to breast cancer. Int J Med Sci. 2021; 18(3):626-638.

36. Šamec $\mathrm{N}$, Zottel A, Videtič Paska A, et al. Nanomedicine and immunotherapy: A step further towards precision medicine for glioblastoma. Molecules. 2020;25(3):490.

37. Wang Y, Sun S, Zhang Z, et al. Nanomaterials for cancer precision medicine. Adv Mater. 2018;30(17):e1705660.

38. Papi M, Palmieri V, Digiacomo L, et al. Converting the personalized biomolecular corona of graphene oxide nanoflakes into a high-throughput diagnostic test for early cancer detection. Nanoscale. 2019;11(32):15339-15346.
39. Godoy-Reyes TM, Costero AM, Gaviña P, et al. Colorimetric detection of normetanephrine, a pheochromocytoma biomarker, using bifunctionalised gold nanoparticles. Anal Chim Acta. 2019;1056:146-152.

40. Zhang F, Stephan SB, Ene CI, et al. Nanoparticles that reshape the tumor milieu create a therapeutic window for effective T-cell therapy in solid malignancies. Cancer Res. 2018;78(13):3718-3730.

41. Song Y, Tang C, Yin C. Combination antitumor immunotherapy with VEGF and PIGF siRNA via systemic delivery of multi-functionalized nanoparticles to tumor-associated macrophages and breast cancer cells. Biomaterials. 2018;185:117-132.

42. Ma W, Zhu D, Li J, et al. Coating biomimetic nanoparticles with chimeric antigen receptor $\mathrm{T}$ cell-membrane provides high specificity for hepatocellular carcinoma photothermal therapy treatment. Theranostics. 2020;10(3):1281-1295

43. Park J, Zhang Y, Saito E, et al. Intravascular innate immune cells reprogrammed via intravenous nanoparticles to promote functional recovery after spinal cord injury. Proc Natl Acad Sci U S A. 2019;116(30):14947-14954.

44. Vhora I, Lalani R, Bhatt P, et al. Lipid-nucleic acid nanoparticles of novel ionizable lipids for systemic BMP-9 gene delivery to bone-marrow mesenchymal stem cells for osteoinduction. Int J Pharm. 2019;563:324-336

45. Patel S, Ryals RC, Weller KK,et al. Lipid nanoparticles for delivery of messenger RNA to the back of the eye. J Control Release. 2019;303:91-100.

46. Tan $\mathrm{T}, \mathrm{Hu} \mathrm{H}$, Wang $\mathrm{H}$, et al. Bioinspired lipoproteins-mediated photothermia remodels tumor stroma to improve cancer cell accessibility of second nanoparticles. Nat Commun. 2019;10(1):3322.

47. Miao L, Li L, Huang $\mathrm{Y}$, et al. Delivery of mRNA vaccines with heterocyclic lipids increases anti-tumor efficacy by STING-mediated immune cell activation. Nat Biotechnol. 2019;37(10):1174-1185.

48. Almeida APB, Damaceno GBR, Carneiro AF, et al. Mucopenetrating lipoplexes modified with PEG and hyaluronic acid for CD44-targeted local siRNA delivery to the lungs. J Biomater Appl. 2019;34(5):617-630.

49. Liu Y, Crowe WN, Wang L, et al. An inhalable nanoparticulate STING agonist synergizes with radiotherapy to confer long-term control of lung metastases. Nat Commun. 2019;10(1):5108.

50. Billingsley MM, Singh N, Ravikumar $\mathrm{P}$, et al. Ionizable lipid nanoparticlemediated mRNA delivery for uman CAR T cell engineering. Nano Lett. 2020;20(3):1578-1589.

51. Knight FC, Gilchuk P, Kumar A, et al. Mucosal immunization with a $\mathrm{pH}$-responsive nanoparticle vaccine induces protective $\mathrm{CD}^{+}$ lung-resident memory T cells. ACS Nano. 2019;13(10):10939-10960.

52. Strand MS, Krasnick BA, Pan $\mathrm{H}$, et al. Precision delivery of RAS-inhibiting siRNA to KRAS driven cancer via peptide-based nanoparticles. Oncotarget. 2019;10(46):4761-4775.

53. Shae D, Becker KW, Christov P, et al. Endosomolytic polymersomes increase the activity of cyclic dinucleotide STING agonists to enhance cancer immunotherapy. Nat Nanotechnol. 2019;14(3):269-278.

54. Zhang L, Wang Y, Yang D, et al. Shape effect of nanoparticles on tumor penetration in monolayers versus spheroids. Mol Pharm. 2019;16(7):2902-2911.

55. Wei L, Zhao $\mathrm{Y}, \mathrm{Hu} \mathrm{X}$, et al. Redox-responsive polycondensate neoepitope for enhanced personalized cancer vaccine. ACS Cent Sci. 2020;6(3):404-412

56. Gao Y, Jia L, Wang Q, et al. $\mathrm{pH} /$ Redox dual-responsive polyplex with effective endosomal escape for codelivery of siRNA and Doxorubicin against drug-resistant cancer cells. ACS Appl Mater Interfaces. 2019;11(18):16296-16310.

57. Lim WQ, Phua SZF, Zhao Y. Redox-responsive polymeric nanocomplex for delivery of cytotoxic protein and chemotherapeutics. ACS Appl Mater Interfaces. 2019;11(35):31638-31648.

58. Shi $\mathrm{H}, \mathrm{Xu} \mathrm{M}$, Zhu J, et al. Programmed co-delivery of platinum nanodrugs and gemcitabine by a clustered nanocarrier for precision chemotherapy for NSCLC tumors. J Mater Chem B. 2020;8(2):332-342.

59. Guo F, Fu Q, Zhou K, et al. Matrix metalloprotein-triggered, cell penetrating peptide-modified star-shaped nanoparticles for tumor targeting and cancer therapy. J Nanobiotechnol. 2020;18(1):48.

60. Xu C, Nam J, Hong $\mathrm{H}$, et al. Positron emission tomography-guided photodynamic therapy with biodegradable mesoporous Silica nanoparticles for personalized cancer immunotherapy. ACS Nano. 2019;13(10):12148-12161.

61. Yeo ELL, Cheah JUJ, Thong PSP, et al. Gold nanorods coated with apolipoprotein E protein corona for drug delivery. ACS Appl Nano Mater. 2019;2:6220-6229.

62. Yi Y, Kim HJ, Zheng M, et al. Glucose-linked sub-50-nm unimer polyion complex-assembled gold nanoparticles for targeted siRNA delivery to 
glucose transporter 1-overexpressing breast cancer stem-like cells. J Control Release. 2019;295:268-277.

63. Zhang L, Su H, Wang $\mathrm{H}$, et al. Tumor chemo-radiotherapy with rod-shaped and spherical gold nano probes: shape and active targeting both matter. Theranostics. 2019;9(7):1893-1908.

64. Ramchandani D, Lee SK, Yomtoubian S, et al. Nanoparticle delivery of miR-708 mimetic impairs breast cancer metastasis. Mol Cancer Ther. 2019;18(3):579-591.

65. $\mathrm{Xu} \mathrm{C}, \mathrm{Gao} \mathrm{F}, \mathrm{Wu} \mathrm{J}$, et al. Biodegradable nanotheranostics with hyperthermia-induced bubble ability for ultrasound imaging-guided chemo-photothermal therapy. Int J Nanomed. 2019;14:7141-7153. 